\title{
PREFERENTIAL DUPLICATION GRAPHS
}

\author{
NETTA COHEN, ${ }^{*}$ University of Leeds \\ JONATHAN JORDAN, ${ }^{* *}$ University of Sheffield \\ MARGARITIS VOLIOTIS, ${ }^{*}$ University of Leeds
}

\begin{abstract}
We consider a preferential duplication model for growing random graphs, extending previous models of duplication graphs by selecting the vertex to be duplicated with probability proportional to its degree. We show that a special case of this model can be analysed using the same stochastic approximation as for vertex-reinforced random walks, and show that 'trapping' behaviour can occur, such that the descendants of a particular group of initial vertices come to dominate the graph.
\end{abstract}

Keywords: Preferential duplication graphs; stochastic approximation; vertex-reinforced random walk

2010 Mathematics Subject Classification: Primary 05C80

Secondary 60G99; 60K35

\section{Introduction}

Many naturally occurring networks ranging from subcellular biological networks to a variety of social networks are believed to grow by processes of vertex duplication. Indeed, graph growing models based on vertex duplications have been the subject of investigation over recent years (see, for example, [1], [5], [6], [7], and [10]). In most of these models vertices are chosen for duplication according to a uniform distribution, while in the model of [5] all vertices are duplicated simultaneously. By contrast, other graph growing algorithms rely on the preferential attachment of new vertices to highly connected existing ones to reproduce the broad, often scalefree, degree distributions (see [4] for a survey) found in many real-world networks. Here we present a generalisation of a duplication graph growing algorithm that is inspired by a 'friendbrings-a-friend' growth process, and reduces to the preferential attachment model in one limit. We call this growth algorithm preferential duplication.

Our model is defined as follows. Let $G_{0}$ be a finite (connected) graph with $n_{0}$ vertices (labelled with the integers $1, \ldots, n_{0}$ ). There are two versions of the model, which we will call the 'false twins' version and the 'true twins' version.

In both models, we construct a sequence of graphs $\left(G_{n}\right)_{n \in \mathbb{N}}$ by a procedure which, to construct $G_{n+1}$ from $G_{n}$, chooses a vertex $v_{n+1}$ of $G_{n}$ with probability proportional to its degree (that is, $v_{n+1}=v$ with probability

$$
\frac{\operatorname{deg}_{G_{n}}(v)}{\sum_{w \in V\left(G_{n}\right)} \operatorname{deg}_{G_{n}}(w)},
$$

Received 6 July 2009; revision received 17 March 2010.

* Postal address: School of Computing, University of Leeds, Leeds, LS2 9JT, UK.

** Postal address: Department of Probability and Statistics, University of Sheffield, Hicks Building, Sheffield, S3 7RH,

UK. Email address: jonathan.jordan@shef.ac.uk 
as in the preferential attachment graph), and duplicates $v_{n+1}$ together with each of its edges with probability $p$, independently of each other. That is, if $v_{n+1}=v$, a new vertex $v^{\prime}$ is added to the graph. An edge exists in $G_{n+1}$ between $v^{\prime}$ and $w$ with probability $p$ if an edge existed in $G_{n}$ between $v$ and $w$, and not otherwise; the existence of edges from $v^{\prime}$ to different neighbours of $v_{n+1}$ is independent. Additionally, in the 'true twins' version of the model only, $v^{\prime}$ is connected to $v$ with probability 1 .

In the remainder of this paper we concentrate on the case $p=1$; we intend to investigate the case $p<1$ in a later paper. We note that the 'true twins' model with $p=0$ becomes the preferential attachment model of [4] with the parameter $m=1$.

The behaviour of preferential duplication graphs bears some resemblance to that of a completely different graph model - that of vertex-reinforced random walks (VRRWs; see [3], [8], and [11]). For a specific duplication event, with $p=1$, the vertex and associated edges duplicated are simply a reproduction of the existing structure of the duplicated vertex. Therefore, it is convenient to collapse the new vertex and its associated edges onto the duplicated vertex and edges, while 'reinforcing' that vertex to indicate that such a collapse has taken place. This process would then be identical to the reinforcement in VRRWs. However, in the former, vertices are selected for duplication from the graph only as a function of their degree, whereas in the latter there is an additional constraint that any two successively reinforced vertices must be neighbours.

We show, in the special case of the preferential duplication model where $p=1$, that a process representing the numbers of descendants of the original vertices becomes 'trapped' on certain subgraphs of the initial graph. In other words, given an initial graph $G_{0}$, we can find subgraphs of $G_{0}$ such that there is a positive probability that after a sufficient number of generations, all new vertices are descendants of vertices in $G_{0}$. Interestingly, such trapping is of the same nature as that which has been shown to occur in VRRWs; our proof method is to show that preferential duplication with $p=1$ has a stochastic approximation equation linking it to the same dynamical system as is linked to VRRWs in [3] and [8]. We conjecture that, with probability 1 , there exists one subgraph that traps the process.

In the 'false twins' case we show in Theorem 2.2 that the trapping subgraphs are of the form $S \cup B$, where $S$ is a complete $m$-partite subgraph of $G_{0}$ satisfying certain conditions and $B$ consists of those vertices with a neighbour in $S$. These trapping subgraphs are the same as those found in a VRRW on the initial graph $G_{0}$. In the 'true twins' case we show in Theorem 2.3 that the trapping subgraphs are of the form $S \cup B$, where $S$ is a maximal clique of $G_{0}$ and $B$ again consists of those vertices with a neighbour in $S$. In both cases any trapping subgraph has positive probability of trapping the process.

The trapping behaviour we find may give insight into the emergence of certain types of structure in a variety of complex systems with similar growth properties. In particular, the principle of a 'friend-brings-a-friend' is commonly used in real-world networks (though rarely with $p=1$ ). Where such growth rules indeed lead to trapping behaviour, we would find that ancestry may be affected more by nuances such as the specific structure of $G_{0}$ than merely the distinctive features of a particular ancestor. Another interesting phenomenon is the symmetry breaking that can occur in this model, with an initial symmetric graph $G_{0}$ that has more than one trapping subgraph. More generally, the fact that trapping appears in two very different models of graph processes suggests that it may be a more universal property of particular classes of systems than previously known. 


\section{The case $p=1$}

We show that the case where $p=1$ (so the new vertex is an exact copy of the vertex it was duplicated from) is closely related to a VRRW (see [3], [8], and [11] for more on VRRWs) on the initial graph.

\subsection{Preliminaries and notation}

Lemma 2.1. In the 'false twins' case, all vertices of $G_{n}$ will have the same set of neighbours in $G_{n}$ as one of the initial vertices $1, \ldots, n_{0}$.

Proof. The new vertex added to $G_{n}$ to form $G_{n+1}$ has the same set of neighbouring vertices in $G_{n+1}$ as the vertex it is a duplicate of. Hence, if two vertices have the same set of neighbours in $G_{n}$ then they will continue to do so in $G_{m}(m>n)$, and so all vertices of $G_{n}$ will have the same set of neighbours in $G_{n}$ as one of the initial vertices $1, \ldots, n_{0}$.

This will also hold in the 'true twins' case if the set of vertices within distance 1 of each vertex (i.e. including the vertex itself) is considered.

We will describe a vertex as being descended from an initial vertex $i$ if it was either duplicated directly from vertex $i$ or duplicated from a vertex descended from vertex $i$. Hence, all vertices descended from vertex $i$ have the same set of neighbours in $G_{n}$ as vertex $i$.

For $n \geq 0$ and $1 \leq i \leq n_{0}$, let $d_{i}^{(n)}$ be the degree of vertex $i$ in $G_{n}$, and let $c_{i}^{(n)}$ be the number of vertices of $G_{n}$ which are descended from vertex $i$ (including vertex $i$ itself). Let $X_{n}$ be a random variable taking values in $\left\{1, \ldots, n_{0}\right\}$, whose value is the original vertex that $v_{n}$ is descended from, so that $c_{X_{n+1}}^{(n+1)}=c_{X_{n+1}}^{(n)}+1$ and $c_{i}^{(n+1)}=c_{i}^{(n)}$ for $i \neq X_{n+1}$.

Let $x_{i}^{(n)}$ be the proportion of vertices of $G_{n}$ descended from vertex $i$,

$$
x_{i}^{(n)}=\frac{c_{i}^{(n)}}{n+n_{0}},
$$

and let $x^{(n)}$ be the vector of proportions, $x^{(n)}=\left(x_{1}^{(n)}, x_{2}^{(n)}, \ldots, x_{n_{0}}^{(n)}\right)$, which can be regarded as an element of the $\left(n_{0}-1\right)$-dimensional simplex

$$
\Delta^{n_{0}-1}=\left\{x \in \mathbb{R}^{n_{0}-1} ; x_{i} \geq 0 \text { for all } i, \sum_{i=1}^{n_{0}-1} x_{i} \leq 1\right\} .
$$

Let $A=\left(a_{i j}\right)_{i, j \in V\left(G_{0}\right)}$ be the adjacency matrix of $G_{0}$, and define a $\sigma$-algebra

$$
\mathcal{F}_{n}=\sigma\left(G_{0}, G_{1}, \ldots, G_{n}\right) .
$$

For the 'false twins' case, let $f(x): \mathbb{R}^{n_{0}} \rightarrow \mathbb{R}^{n_{0}}$ be a function with coordinates given by

$$
f_{i}(x)=\frac{x_{i} \sum_{j=1}^{n_{0}} a_{i j} x_{j}}{\sum_{k=1}^{n_{0}} x_{k} \sum_{j=1}^{n_{0}} a_{k j} x_{j}}=\frac{x_{i}(A x)_{i}}{x^{\top} A x},
$$

and let $F(x)=f(x)-x$. Following [3], for $x \in \Delta^{n_{0}-1}$, we write $N_{i}(x)=(A x)_{i}$ and $H(x)=\sum_{i=1}^{n_{0}} x_{i} N_{i}(x)=x^{\top} A x$, so that

$$
f_{i}(x)=\frac{x_{i} N_{i}(x)}{H(x)} \quad \text { and } \quad F_{i}(x)=\frac{x_{i}\left(N_{i}(x)-H(x)\right)}{H(x)} .
$$

Similarly, for the 'true twins' case, define $\hat{N}_{i}(x)=[(A+I) x]_{i}$ (where $I$ is the $n_{0} \times n_{0}$ identity matrix), $\hat{H}(x)=x^{\top}(A+I) x, \hat{f}_{i}(x)=x_{i} \hat{N}_{i}(x) / \hat{H}(x)$, and $\hat{F}(x)=\hat{f}(x)-x$.

For $i \in\left\{1, \ldots, n_{0}\right\}$, let $\iota(i)$ be the unit vector in $\mathbb{R}^{n_{0}}$ with 1 in position $i$ and 0 s elsewhere. 


\subsection{Stochastic approximation and analysis of attractors}

Theorem 2.1. In the 'false twins' case, the sequence $\left(x^{(n)}\right)_{n \in \mathbb{N}}$ satisfies the stochastic approximation equation

$$
x^{(n+1)}-x^{(n)}=\frac{1}{n+n_{0}+1} F\left(x^{(n)}\right)+\varepsilon^{(n+1)},
$$

with $\mathrm{E}\left(\varepsilon^{(n+1)} \mid \mathcal{F}_{n}\right)=0$ and where $\varepsilon^{(n+1)}\left(n+n_{0}+1\right)$ is bounded.

In the 'true twins' case, the sequence satisfies a different stochastic approximation equation,

$$
x^{(n+1)}-x^{(n)}=\frac{1}{n+n_{0}+1} \hat{F}\left(x^{(n)}\right)+\varepsilon^{(n+1)}+R^{(n+1)},
$$

with $\mathrm{E}\left(\varepsilon^{(n+1)} \mid \mathcal{F}_{n}\right)=0$, where $\varepsilon^{(n+1)}\left(n+n_{0}+1\right)$ is bounded and the remainder term $R^{(n)}=$ $O\left(n^{-2}\right)$.

Proof. The graph $G_{n}$ has $n+n_{0}$ vertices. The new vertex $v_{n+1}$ will be descended from vertex $i$ if and only if the selected vertex $v_{n+1}$ is. Hence, in the 'false twins' case,

$$
c_{i}^{(n+1)}= \begin{cases}c_{i}^{(n)}+1 & \text { with probability } \frac{c_{i}^{(n)} d_{i}^{(n)}}{\sum_{k=1}^{n_{0}} c_{k}^{(n)} d_{k}^{(n)}}, \\ c_{i}^{(n)} & \text { with probability } 1-\frac{c_{i}^{(n)} d_{i}^{(n)}}{\sum_{k=1}^{n_{0}} c_{k}^{(n)} d_{k}^{(n)}} .\end{cases}
$$

Let $a_{i j}, 1 \leq i, j \leq n_{0}$, be the entries of the adjacency matrix of $G_{0}$. Then $d_{i}^{(n)}=$ $\sum_{j=1}^{n_{0}} a_{i j} c_{j}^{(n)}$, so we can rewrite the above as

$$
c_{i}^{(n+1)}= \begin{cases}c_{i}^{(n)}+1 & \text { with probability } \frac{c_{i}^{(n)} \sum_{j=1}^{n_{0}} a_{i j} c_{j}^{(n)}}{\sum_{k=1}^{n_{0}} c_{k}^{(n)} \sum_{j=1}^{n_{0}} a_{k j} c_{j}^{(n)}}, \\ c_{i}^{(n)} & \text { with probability } 1-\frac{c_{i}^{(n)} \sum_{j=1}^{n_{0}} a_{i j} c_{j}^{(n)}}{\sum_{k=1}^{n_{0}} c_{k}^{(n)} \sum_{j=1}^{n_{0}} a_{k j} c_{j}^{(n)}} .\end{cases}
$$

Hence, this can be treated as a generalisation of the Pólya urn model where category $i$ is chosen with probability

$$
\frac{c_{i}^{(n)} \sum_{j=1}^{n_{0}} a_{i j} c_{j}^{(n)}}{\sum_{k=1}^{n_{0}} c_{k}^{(n)} \sum_{j=1}^{n_{0}} a_{k j} c_{j}^{(n)}}=f_{i}\left(x^{(n)}\right)
$$

instead of with probability simply proportional to $c_{i}^{(n)}$.

We see that

$$
x_{i}^{(n+1)}-x_{i}^{(n)}=\frac{1}{n+n_{0}+1}\left(1-x_{i}^{(n)}\right) \mathbf{1}_{\left\{X_{n+1}=i\right\}}-\frac{1}{n+n_{0}+1} x_{i}^{(n)}\left(1-\mathbf{1}_{\left\{X_{n+1}=i\right\}}\right),
$$

and, hence,

$$
x^{(n+1)}-x^{(n)}=\frac{1}{n+n_{0}+1}\left(\iota\left(X_{n+1}\right)-x^{(n)}\right) .
$$

Taking conditional expectations,

$$
\mathrm{E}\left(x^{(n+1)}-x^{(n)} \mid \mathcal{F}_{n}\right)=\frac{1}{n+n_{0}+1}\left(f\left(x^{(n)}\right)-x^{(n)}\right) .
$$


Letting

$$
\varepsilon^{(n+1)}=\frac{1}{n+n_{0}+1}\left(\iota\left(X_{n+1}\right)-f\left(x^{(n)}\right)\right),
$$

we conclude that $\mathrm{E}\left(\varepsilon^{(n+1)} \mid \mathscr{F}_{n}\right)=0$ and that $\varepsilon^{(n+1)}\left(n+n_{0}+1\right)$ is bounded, giving the result.

In the 'true twins' case,

$$
d_{i}^{(n)}=\sum_{j=1}^{n_{0}} a_{i j} c_{j}^{(n)}+c_{i}^{(n)}-1 .
$$

So the probability that some vertex descended from vertex $i$ is chosen for duplication is

$$
\begin{aligned}
p_{i}^{(n)} & =\frac{c_{i}^{(n)}\left(\sum_{j=1}^{n_{0}} a_{i j} c_{j}^{(n)}+c_{i}^{(n)}-1\right)}{\sum_{k=1}^{n_{0}} c_{k}^{(n)} \sum_{j=1}^{n_{0}}\left(a_{k j} c_{j}^{(n)}+c_{k}^{(n)}-1\right)} \\
& =\frac{x_{i}^{(n)}\left(\sum_{j=1}^{n_{0}} a_{i j} x_{j}^{(n)}+x_{i}^{(n)}-1 /\left(n+n_{0}\right)\right)}{\sum_{k=1}^{n_{0}} x_{k}^{(n)} \sum_{j=1}^{n_{0}}\left(a_{k j} x_{j}^{(n)}+x_{k}^{(n)}-1 /\left(n+n_{0}\right)\right)},
\end{aligned}
$$

so

$$
\begin{aligned}
p_{i}^{(n)} & =\frac{x_{i}^{(n)}\left(\hat{N}_{i}\left(x^{(n)}\right)-1 /\left(n+n_{0}\right)\right)}{\hat{H}\left(x^{(n)}\right)-1 /\left(n+n_{0}\right)} \\
& =\frac{x_{i}^{(n)} \hat{N}_{i}\left(x^{(n)}\right)}{\hat{H}\left(x^{(n)}\right)}+\frac{x_{i}^{(n)}\left(\hat{N}_{i}\left(x^{(n)}\right)-\hat{H}\left(x^{(n)}\right)\right)}{\hat{H}\left(x^{(n)}\right)\left(\hat{H}\left(x^{(n)}\right)\left(n+n_{0}\right)-1\right)} \\
& =\hat{f}_{i}\left(x^{(n)}\right)+O\left(n^{-1}\right) .
\end{aligned}
$$

Hence, stochastic approximation theory [2], [9] relates the behaviour of $x^{(n)}$ to the behaviour of the continuous-time dynamical system given by the function $F(x)$. This is the same stochastic approximation as occurs for vertex-reinforced random walks in [3] and [8].

An attractor for a flow $\Phi$ on a metric space $(M, d)$ is defined (e.g. in [2]) to be a subset $A \subseteq M$ which is invariant under $\Phi$ and has a neighbourhood $W$ such that $d\left(\Phi_{t} x, A\right) \rightarrow 0$ as $t \rightarrow \infty$ uniformly for $x \in W$.

In [3], a stable equilibrium for the dynamical system of interest is defined as being an equilibrium where all eigenvalues of the Jacobian matrix are nonpositive. This does not necessarily imply that the stable equilibrium is in an attractor.

As $H$ is a Lyapunov function for the stochastic approximation, and letting $L(x)$ be the limit set of the process $\left(x^{(n)}\right)_{n \in \mathbb{N}}$, we can conclude the following.

Corollary 2.1. If $A$ is an attractor of the continuous-time dynamical system given by the function $F(x)$ (or $\hat{F}(x))$, then $\mathrm{P}(L(x) \subseteq A)>0$. Furthermore, $L(x)$ consists of equilibria for the dynamical system, and $H\left(x^{(n)}\right)$ (or $\left.\hat{H}\left(x^{(n)}\right)\right)$ converges as $n \rightarrow \infty$.

Proof. The first statement follows from Theorem 7.3 of [2] and the second statement follows from Proposition 6.4 of [2].

We now discuss the attractors and stable equilibria for the dynamical system in the 'false twins' case. First consider the case where $G_{0}$ is a complete $m$-partite graph. In this case vertices which belong to the same part have the same neighbours and so are indistinguishable, so we can just consider the case where $G_{0}$ is a complete graph on $m$ vertices. The convergence of the stochastic approximation in this case is discussed in [8]. 
We now consider finding attractors for the dynamical system in more general graphs. Consider the case where $G_{0}$ contains a subgraph consisting of a 'core' $S$ and its outer boundary $B$, $S$ being a complete $m$-partite graph, $S=V_{1} \cup V_{2} \cup \cdots \cup V_{m}$, and $B$ consisting of exactly those vertices which are outside $S$ but have a neighbour in $S$. We have already dealt with the case where $S$ is the whole graph above, so without loss of generality assume that $n_{0} \notin S$. In [11] such a subgraph is defined to be a trapping subgraph if, for any vertex $v$ in $B$, two criteria are met:

1. there is at least one part of $S, V_{i}$, such that $v$ is not connected to $V_{i}$;

2. there is at least one vertex in $x^{\prime} \in S \backslash V_{i}$ such that $v$ is not connected to $x^{\prime}$.

The following result shows that trapping subgraphs (under the definition in [11]) produce attractors of the dynamical system.

Proposition 2.1. Let $S$ be the core of a trapping subgraph, and let $y \in \Delta^{n_{0}-1}$ be such that $\sum_{i \in V_{j}} y_{i}=1 / m$ and that $y_{i}=0$ if $i \notin S$, i.e. y represents a proportion $1 / m$ of the vertices being in each part of the m-partite graph, and a proportion 0 outside the graph. The set of points of this form is an attractor for the dynamical system driven by $F$.

Proof. It is fairly easy to see that $y$ is a fixed point of $F$ : the definition implies that $H(y)=$ $(m-1) / m$ and that $N_{i}(y)=H(y)$ for all $i \in S$. It remains to prove that the set of fixed points of this form is an attractor for the dynamical system. To do this, we will evaluate the partial derivatives $d_{i j}=\partial F_{i} / \partial x_{j}$ at the fixed point $y$, and show that the eigenvalues of the resulting Jacobian are at most 0 , and that the eigenspace corresponding to the eigenvalue 0 is contained within the set of fixed points.

Using the fact that $\sum_{i=1}^{n_{0}} x_{i}^{(n)}=1$, we write $x_{n_{0}}=1-\sum_{i=1}^{n_{0}-1} x_{i}$ and treat $f$ as a function from $\Delta^{n_{0}-1}$ to itself. Hence, we can rewrite the components of $f$ :

$$
f_{i}(x)=\frac{x_{i} \sum_{j=1}^{n_{0}-1}\left(a_{i j}-a_{i, n_{0}}\right) x_{j}+x_{i} a_{i, n_{0}}}{\sum_{k=1}^{n_{0}-1}\left(\sum_{j=1}^{n_{0}-1}\left(a_{k j}-2 a_{k, n_{0}}\right) x_{k} x_{j}+2 a_{k, n_{0}} x_{k}\right)} .
$$

(We assume that $G_{0}$ has no loops, so $a_{i i}=0$ for all $i$.)

Differentiating and substituting the above values for the $x_{i}$ at the fixed point, we find that

$$
\begin{aligned}
d_{i j}= & \left.\frac{\partial F_{i}}{\partial x_{j}}\right|_{x=y} \\
& = \begin{cases}-\frac{m a_{i, n_{0}} y_{i}}{m-1}-2 y_{i}+\frac{2 m y_{i}}{m-1} \sum_{k \in S} a_{k, n_{0}} y_{k}, & i=j \in S, \\
\frac{m a_{i j} y_{i}}{m-1}-\frac{m a_{i, n_{0}} y_{i}}{m-1}-2 y_{i}+\frac{2 m y_{i}}{m-1} \sum_{k \in S} a_{k, n_{0}} y_{k}, & i \neq j, i, j \in S, \\
\frac{m a_{i j} y_{i}}{m-1}-\frac{m a_{i, n_{0}} y_{i}}{m-1}-\frac{2 m y_{i}}{m-1} \sum_{k \in S} a_{k j} y_{k}+\frac{2 m y_{i}}{(m-1)} \sum_{k \in S} a_{k, n_{0}} y_{k}, & i \in S, j \notin S, \\
\frac{m}{m-1} \sum_{k \in S} a_{i k} y_{k}-1, & i=j \notin S, \\
0, & i \notin S, j \neq i .\end{cases}
\end{aligned}
$$


The off-diagonal zero entries where $i \notin S$ mean that the eigenvalues of this matrix are the eigenvalues of the matrix obtained by restricting to the rows and columns corresponding to $S$, together with the diagonal entries

$$
\eta_{i}=\frac{m}{m-1} \sum_{k \in S} a_{i k} y_{k}-1, \quad i \notin S .
$$

So we need to find the eigenvalues of the $|S| \times|S|$ matrix $D$ with entries

$$
d_{i j}=\left.\frac{\partial F_{i}}{\partial x_{j}}\right|_{x=y}= \begin{cases}-\frac{m a_{i, n_{0}} y_{i}}{m-1}-2 y_{i}+\frac{2 m y_{i}}{m-1} \sum_{k \in S} a_{k, n_{0}} y_{k}, & i=j \in S, \\ \frac{m a_{i j} y_{i}}{m-1}-\frac{m a_{i, n_{0}} y_{i}}{m-1}-2 y_{i}+\frac{2 m y_{i}}{(m-1)} \sum_{k \in S} a_{k, n_{0}} y_{k}, & i \neq j, i, j \in S .\end{cases}
$$

Label the parts of the complete $m$-partite graph $S$ as $1, \ldots, m$, and let $p(i)$ be the part containing vertex $i$. Then, given a set of constants $\alpha_{1}, \alpha_{2}, \ldots, \alpha_{m}$ with $\sum_{k=1}^{m} \alpha_{k}=0$, define a vector $v \in \mathbb{R}^{|S|}$ by $v_{i}=y_{i} \alpha_{p(i)}$. Then, as $a_{i j}$ is 1 if $p(i) \neq p(j)$ and 0 otherwise,

$$
(D v)_{i}=\frac{m y_{i}}{m-1} \sum_{\{j: p(j) \neq p(i)\}} y_{j} \alpha_{p(j)}=\frac{y_{i}}{m-1}\left(-\alpha_{p(i)}\right)=-\frac{1}{m-1} v_{i},
$$

so this gives an eigenspace of dimension $m-1$ with eigenvalue $-1 /(m-1)$.

Now let $w$ be a vector with $w_{j}=1$ for all $j$. Then

$$
\begin{aligned}
\left(w^{\top} S\right)_{j} & =\sum_{i \in S}\left(\frac{m a_{i j} y_{i}}{m-1}-\frac{m a_{i, n_{0}} y_{i}}{m-1}-2 y_{i}+\frac{2 y_{i}}{m-1} \sum_{k \in S} m a_{k, n_{0}} y_{k}\right) \\
& =1-\frac{1}{m-1} \sum_{i \in S} m a_{i, n_{0}} y_{i}-2+\frac{2}{m-1} \sum_{i \in S} m a_{i, n_{0}} y_{i} \\
& =\frac{m}{m-1} \sum_{i \in S} a_{i, n_{0}} y_{i}-1,
\end{aligned}
$$

so there is a dimension 1 eigenspace with eigenvalue

$$
\eta_{n_{0}}=\frac{m}{m-1} \sum_{i \in S} a_{i, n_{0}} y_{i}-1 .
$$

If $p(i)=p(j), a_{i j}=0$, so rows $i$ and $j$ of $D$ are identical. Hence, each part $k$ gives an eigenspace with eigenvalue 0 and dimension $\left|V_{k}\right|-1$. The eigenspace of the zero eigenvalues is in the direction where $F$ is constant.

We now need to consider the eigenvalues $\eta_{i}$ for $i \notin S$. If our subgraph is a trapping subgraph then this ensures that, as $i$ is outside $S$,

$$
\sum_{k \in S} a_{i k} y_{k}<\frac{m-1}{m}
$$

and so these eigenvalues are negative for all choices of $y_{k}, k \in S$. Hence, in this case all the eigenvalues are negative or 0 , and the eigenspace of the zero eigenvalues is in the direction where $F$ is constant, so the set of fixed points is an attractor. 
A slightly weaker condition than that of a trapping subgraph in [11] arises if it is possible to find an equilibrium $y$ with support $S$ such that

$$
\sum_{k \in S} a_{i k} y_{k}<\frac{m-1}{m},
$$

or, equivalently,

$$
N_{i}(y)<H(y)
$$

simultaneously for all $i \notin S$. In this case there is a region of the family of fixed points where all the eigenvalues are negative or 0 , and the eigenspace of the zero eigenvalues is in the direction where $F$ is constant, but this does not apply throughout the family of fixed points, so the family is not an attractor according to the standard definition. In what follows, we will extend the definition of a trapping subgraph from that in [11] by including those where it is possible to find such an equilibrium $y$.

For example, the simplest case where this arises is the graph with five vertices:

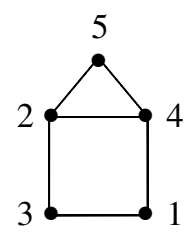

This graph contains a trapping subgraph according to the definition in [11], where $S$ is the triangle formed by vertices $\{2,4,5\}$. However, if $S$ is the bipartite graph $\{1,2\} \cup\{3,4\}$ and $y_{2}+y_{4}<\frac{1}{2}$, then the eigenvalues other than those within the family of fixed points are all negative.

In the context of VRRWs, it was shown in [3] that any stable equilibrium $y$ of the dynamical system has support consisting of a complete $m$-partite graph $S$, and that the condition mentioned above that (2.1) is satisfied simultaneously for all $i \notin S$ implies that there is positive probability of VRRWs being trapped in a neighbourhood of $y$.

The results for VRRWs in [3] also show that stable equilibria of the dynamical system driven by $\hat{F}$, which appears in the 'true twins' case, are localised on cliques of the original graph $G_{0}$ : if $S$ is a clique of $G_{0}$ then any $y$ with $\sum_{i \in S} y_{i}=1$ is a stable equilibrium. The condition that $\hat{N}_{i}(y)<\hat{H}(y)$ simultaneously for all $i \notin S$ implies that $S$ is not contained within a larger clique.

\subsection{Convergence to stable equilibria}

In this section we show that in the 'false twins' case any trapping subgraph (in the weaker sense described above) has a positive probability of trapping the process $x^{(n)}$. Throughout the proofs, similar arguments can be applied in the 'true twins' case to show that any clique of $G_{0}$ which is not contained within a larger clique can trap the process. The method, and the proofs of Lemmas 2.2 and 2.3, are based on those used for VRRWs in [3].

The following definitions and notation follow [3]. Let $S$ be a complete $k$-partite subgraph of $G_{0}$ with outer boundary $B$. Let $\&$ consist of elements of $\Delta^{n_{0}-1}$ whose support is $S$, and let $\delta^{\prime}$ consist of elements of $\Delta^{n_{0}-1}$ which are nonzero at all elements of $S$. Let $\mathcal{L}(U)$ be the event that $x^{(n)}$ converges to a stable equilibrium $x^{(\infty)} \in \delta \cap U$. 
Given $q \in \S$, define the entropy function

$$
V_{q}(y)= \begin{cases}-\sum_{i \in S} q_{i} \log \left(\frac{y_{i}}{q_{i}}\right)+2 \sum_{i \notin S} y_{i}, & y \in \AA^{\prime} \\ \infty, & \text { otherwise }\end{cases}
$$

We define two types of balls around $q$, one based on the entropy function,

$$
B_{V_{q}}(r)=\left\{y \in \Delta^{n_{0}-1}: V_{q}(y)<r\right\}
$$

and one based on the $\infty$-norm,

$$
B_{\infty}(q, r)=\left\{y \in \Delta^{n_{0}-1}:\|y-q\|_{\infty}<r\right\}
$$

As stated in [3], there are increasing continuous functions $u_{1, q}$ and $u_{2, q}: \mathbb{R}^{+} \rightarrow \mathbb{R}^{+}$such that $u_{1, q}(0)=0$ and $u_{2, q}(0)=0$, and, for all $r>0, B_{\infty}\left(q, u_{1, q}(r)\right) \subset B_{V_{q}}(r) \subset B_{\infty}\left(q, u_{2, q}(r)\right)$.

Let

$$
\zeta_{i}^{(n+1)}= \begin{cases}\frac{\varepsilon_{i}^{(n+1)}}{x_{i}^{(n)}}, & i \in S, x_{j}^{(n)} \neq 0 \text { for all } j \in S, \\ 0, & \text { otherwise. }\end{cases}
$$

Still following [3], for $q, z \in \Delta^{n_{0}-1}$, let

$$
\begin{aligned}
I_{q}(z) & =-\sum_{i \in S} q_{i}\left(N_{i}(z)-H(z)\right)+2 \sum_{i \notin S} z_{i}\left(N_{i}(z)-H(z)\right) \\
& =-H(z)\left(-\sum_{i \in S} q_{i} \frac{F_{i}(z)}{z_{i}}+2 \sum_{i \notin S} F_{i}(z)\right) .
\end{aligned}
$$

In the following lemma, this quantity will be related to the increment in entropy relative to $q$ between $x^{(n)}$ and $x^{(n+1)}$.

The following lemma corresponds to Lemma 5 of [3], with a virtually identical proof.

Lemma 2.2. ([3, Lemma 5].) Let $q \in \delta$ be a stable equilibrium of the dynamical system with $N_{i}(q)<H(q)$ for all $i \in B$. There exists $\varepsilon$ such that if $n$ is large enough and $x^{(n)} \in B_{V_{q}}(\varepsilon)$, then

$$
V_{q}\left(x^{(n+1)}\right)-V_{q}\left(x^{(n)}\right)=\frac{I_{q}\left(x^{(n)}\right)}{\left(n+n_{0}+1\right) H\left(x^{(n)}\right)}-\left\langle q, \zeta^{(n+1)}\right\rangle+2 \sum_{i \notin S} \varepsilon_{i}^{(n+1)}+O\left(\frac{1}{\left(n+n_{0}\right)^{2}}\right),
$$

and, furthermore,

$$
I_{q}\left(x^{(n)}\right) \leq-\left(H(q)-H\left(x^{(n)}\right)+C_{1} \sum_{i \notin S} x_{i}^{(n)}\right)
$$

for a positive constant $C_{1}$. 
Proof. We have

$$
\begin{aligned}
& V_{q}\left(x^{(n+1)}\right)-V_{q}\left(x^{(n)}\right) \\
&=-\sum_{i \in S} q_{i}\left(\log \left(\frac{x_{i}^{(n+1)}}{q_{i}}\right)-\log \left(\frac{x_{i}^{(n)}}{q_{i}}\right)\right)+2 \sum_{i \notin S}\left(x_{i}^{(n+1)}-x_{i}^{(n)}\right) \\
&=-\sum_{i \in S} q_{i} \frac{x_{i}^{(n+1)}-x_{i}^{(n)}}{x_{i}^{(n)}}+2 \sum_{i \notin S}\left(x_{i}^{(n+1)}-x_{i}^{(n)}\right)+O\left(\frac{1}{\left(n+n_{0}\right)^{2}}\right) \\
&=-\sum_{i \in S} q_{i} \frac{F_{i}\left(x^{(n)}\right)}{\left(n+n_{0}+1\right) x_{i}^{(n)}}-\sum_{i \in S} q_{i} \zeta_{i}^{(n+1)}+2 \sum_{i \notin S}\left(\frac{F_{i}\left(x^{(n)}\right)}{n+n_{0}+1}+\varepsilon_{i}^{(n+1)}\right) \\
&+ O\left(\frac{1}{\left(n+n_{0}\right)^{2}}\right) \\
&= \frac{I_{q}\left(x^{(n)}\right)}{\left(n+n_{0}+1\right) H\left(x^{(n)}\right)}-\left\langle q, \zeta^{(n+1)}\right\rangle+2 \sum_{i \notin S} \varepsilon_{i}^{(n+1)}+O\left(\frac{1}{\left(n+n_{0}\right)^{2}}\right) .
\end{aligned}
$$

(by Taylor's expansion)

For the inequality for $I_{q}\left(x^{(n)}\right)$, observe that

$$
\sum_{i \in S} q_{i} N_{i}(z)=\sum_{i \in G} q_{i} N_{i}(z)=\sum_{i \in G} z_{i} N_{i}(q)=H(q)+\sum_{i \in B} z_{i}\left(N_{i}(q)-H(q)\right),
$$

by the definition of $N_{i}$ and the fact that $q$ is an equilibrium. So

$$
I_{q}(z)=H(z)-H(q)+\sum_{i \in B} z_{i}\left(2\left(N_{i}(z)-H(z)\right)-\left(N_{i}(q)-H(q)\right)\right),
$$

so the inequality is satisfied if we choose $\varepsilon$ small enough that if $z \in B_{V_{q}}(\varepsilon), 2\left(N_{i}(z)-H(z)\right)-$ $\left(N_{i}(q)-H(q)\right)<-C_{1}$ for all $i \notin S$.

Lemma 2.3. ([3, Lemma 7].) Let $q \in \&$ be a stable equilibrium of the dynamical system with $N_{i}(q)<H(q)$ for all $i \in B$. For sufficiently small $\varepsilon$ and sufficiently large $n$, if $x^{(n)} \in B_{V_{x}}(\varepsilon / 2)$,

$$
\mathrm{P}\left(\mathcal{L}\left(B_{V_{q}}(\varepsilon)\right) \mid \mathcal{F}_{n}\right) \geq 1-\exp \left(-\varepsilon^{2} C_{2}\left(n+n_{0}\right)\right) .
$$

Proof. The proof follows that of Lemma 7 of [3].

Fix $\varepsilon$ small enough that, for all $x \in B_{V_{q}}(\varepsilon), x_{i} \geq \alpha$ for all $i \in S$ and some positive $\alpha$. Define martingales $\left(A_{k}\right)_{k \geq n},\left(B_{k}\right)_{k \geq n}$, and $\left(\kappa_{k}\right)_{k \geq n}$ by $A_{n}=B_{n}=\kappa_{n}=0$, and, for $k>n$,

$$
\begin{aligned}
A_{k} & =\sum_{j=n+1}^{k} \zeta^{(j)} \mathbf{1}_{\left\{V_{q}\left(x^{(j-1)}\right)<\varepsilon\right\}}, \\
B_{k} & =\sum_{j=n+1}^{k} \sum_{i \in B} \varepsilon_{i}^{(j)} \mathbf{1}_{\left\{V_{q}\left(x^{(j-1)}\right)<\varepsilon\right\}}, \\
\kappa_{k} & =-\left\langle q, A_{k}\right\rangle+2 B_{k} .
\end{aligned}
$$


By martingale convergence, all three converge almost surely and in $L^{2}$, and as increments of $\kappa$ have moduli at most $C_{3} /\left(k+n_{0}\right)$ for some constant $C_{3}$,

$$
\mathrm{E}\left(\exp \left(\theta\left(\kappa_{k}-\kappa_{k-1}\right) \mid \mathcal{F}_{k-1}\right)\right) \leq \exp \left(\frac{C_{3}^{2}}{2} \frac{\theta^{2}}{\left(k+n_{0}\right)^{2}}\right) .
$$

As $\left(\kappa_{k}\right)_{k \geq n}$ is a martingale, $\left(\exp \left(\kappa_{k}\right)\right)_{k \geq n}$ is a submartingale, so Doob's submartingale inequality implies that

$$
\mathrm{P}\left(\sup _{k \geq n} \kappa_{k} \geq c \mid \mathcal{F}_{n}\right) \leq \mathrm{e}^{-\theta c} \mathrm{E}\left(\mathrm{e}^{\theta \kappa_{\infty}} \mid \mathcal{F}_{n}\right) \leq \exp \left(-\theta c+\frac{\theta^{2} C_{3}^{2}}{2\left(n+n_{0}\right)}\right),
$$

so if $\theta=c\left(n+n_{0}\right) / C_{3}^{2}$ then

$$
\mathrm{P}\left(\sup _{k \geq n} \kappa_{k} \geq c \mid \mathcal{F}_{n}\right) \leq \exp \left(-\frac{c^{2}}{2 C_{3}^{2}}\left(n+n_{0}\right)\right) .
$$

Let $\Upsilon$ be the event that $\sup _{k \geq n} \kappa_{k}<\varepsilon / 4$; then

$$
\mathrm{P}\left(\Upsilon \mid \mathcal{F}_{n}\right) \geq 1-\exp \left(-\varepsilon^{2} C_{2}\left(n+n_{0}\right)\right)
$$

for a new constant $C_{2}$.

Lemma 2.2 implies that

$$
V_{q}\left(x^{(k)}\right)-V_{q}\left(x^{(n)}\right) \leq \kappa_{k}+\frac{\varepsilon}{4}
$$

if $n$ is large enough (as in [3], we use the fact that Lemma 4 of [3] implies that $H(q)-H\left(x^{(n)}\right) \geq$ 0 if $x^{(n)} \in B_{V_{q}}(\varepsilon)$ for small enough $\varepsilon$ ). Hence, on $\Upsilon, V_{q}\left(x^{(k)}\right)<\varepsilon$ for all $k \geq n$.

Lemma 2.2 now implies that, as $\kappa_{k}$ converges as $k \rightarrow \infty, \liminf _{k \rightarrow \infty}\left(-I_{q}\left(x^{(k)}\right)=0\right.$ (otherwise $V_{q}\left(x^{(k)}\right) \rightarrow-\infty$, but $V_{q}(x)>0$ for all $x$ ) and so

$$
\liminf _{k \rightarrow \infty}\left(H(q)-H\left(x^{(k)}\right)+C_{1} \sum_{i \notin S} x_{i}^{(k)}\right)=0 .
$$

Hence, there exists a subsequence $\left(j_{k}\right)_{\{k \geq 0\}}$ with

$$
\lim _{k \rightarrow \infty} H\left(x^{\left(j_{k}\right)}\right)=H(q)
$$

and

$$
\lim _{k \rightarrow \infty} \sum_{i \notin S} x_{i}^{\left(j_{k}\right)}=0
$$

As in [3], we identify an accumulation point $r$ of $\left(x^{\left(j_{k}\right)}\right)_{\{k \geq 0\}}$, which will have $H(r)=H(q)$ and, hence (by the lemmas in [3]), be a stable equilibrium if $\varepsilon$ is small enough.

Redefine the martingale $\left(\kappa_{k}\right)_{k \geq n}$ in terms of $r$ instead of $q$, and let $j_{k}$ be far enough along this subsequence that $V_{r}\left(x^{\left(j_{k}\right)}\right)<\varepsilon / 2$ and $\sup _{k \geq j_{k}}\left|\kappa_{k}-\kappa_{j}\right|<\varepsilon / 4$. Then Lemma 2.2 implies that, for $j^{\prime}>j>j_{k}$,

$$
V_{r}\left(x^{\left(j^{\prime}\right)}\right) \leq V_{r}\left(x^{(j)}\right)+\sup _{k>j}\left|\kappa_{k}-\kappa_{j}\right|+\frac{C_{4}}{j} .
$$


As $\liminf \inf _{j \rightarrow \infty} V_{r}\left(x^{(j)}\right)=0$ and $\lim _{j \rightarrow \infty}\left(\sup _{k>j}\left|\kappa_{k}-\kappa_{j}\right|+C_{4} / j\right)=0$, we have

$$
V_{r}\left(x^{(n)}\right) \rightarrow \lim _{k \rightarrow \infty} V_{r}\left(x^{\left(j_{k}\right)}\right)=0
$$

and so $x^{(n)} \rightarrow r$.

Lemma 2.4. Assume that, for a given vertex $i, N_{i}\left(x^{(n)}\right) / H\left(x^{(n)}\right)$ converges to $\lambda_{i} \in(0, \infty)$. Then, for $i \in B, c_{i}^{(n)} / n^{\lambda_{i}}$ converges to a limit in $(0, \infty)$ almost surely.

Proof. Let

$$
Y_{i}^{(n)}=\sum_{k=1}^{n} \frac{\mathbf{1}_{\left\{X_{k}=i\right\}}}{c_{i}^{(k-1)}}
$$

and let

$$
M_{i}^{(n)}=Y_{i}^{(n)}-\sum_{k=1}^{n} \frac{N_{i}\left(x^{(k-1)}\right)}{H\left(x^{(k-1)}\right)\left(k+n_{0}\right)} .
$$

Then

$$
\mathrm{E}\left(Y_{i}^{(n+1)}-Y_{i}^{(n)} \mid \mathcal{F}_{n}\right)=\frac{N_{i}\left(x^{(n)}\right)}{H\left(x^{(n)}\right)\left(n+n_{0}\right)},
$$

so $\left(M_{i}^{(n)}\right)_{n \geq 1}$ is a martingale.

Now,

$$
\begin{aligned}
\mathrm{E}\left(\sum_{n=1}^{\infty}\left(M_{i}^{(n)}-M_{i}^{(n-1)}\right)^{2}\right) & =\mathrm{E}\left(\sum_{n=1}^{\infty}\left(\frac{\mathbf{1}_{\left\{X_{n}=i\right\}}}{c_{i}^{(n-1)}}-\frac{N_{i}\left(x^{(n-1)}\right)}{H\left(x^{(n-1)}\right)\left(n+n_{0}\right)}\right)^{2}\right) \\
& \leq \mathrm{E}\left(\sum_{n=1}^{\infty}\left(\frac{\mathbf{1}_{\left\{X_{n}=i\right\}}}{c_{i}^{(n-1)}}\right)^{2}+\left(\frac{N_{i}\left(x^{(n-1)}\right)}{H\left(x^{(n-1)}\right)\left(n+n_{0}\right)}\right)^{2}\right) \\
& \leq \infty
\end{aligned}
$$

so martingale convergence implies that

$$
\log c_{i}^{(n)} \equiv Y_{i}^{(n)} \equiv \lambda_{i} \log n,
$$

giving the result.

Let $\mathcal{R}_{n, k}$ be the range of the process $\left(X_{j}\right)_{j \in \mathbb{N}}$ between times $n$ and $k$, and let $\mathcal{R}_{n, \infty}$ be the range of the process $\left(X_{j}\right)_{j \in \mathbb{N}}$ for times $j \geq n$.

We now combine our results.

Theorem 2.2. In the 'false twins' case, let $G_{0}$ contain a complete m-partite graph $S$ with outer boundary $B$ such that there exists a stable equilibrium $q$ of the dynamical system driven by $F$ with support $S$ and with $N_{i}(q)<H(q)$ for all $i \in B$. Then, with positive probability, for some stable equilibrium $r \in B_{V_{q}}(\varepsilon)$ with support $S$,

1. $x^{(n)} \rightarrow r$;

2. for $i \in B, c_{i}^{(n)} / n^{N_{i}(r) / H(r)}$ converges to a limit in $(0, \infty)$ almost surely;

3. for some (random) time $n, \mathcal{R}_{n, \infty}=S \cup B$. 
Furthermore, if there is a stable equilibrium $r$ in the limit set $L(x)$ of $\left(x^{(n)}\right)_{n \in \mathbb{N}}$ with support $S$ and with $N_{i}(r)<H(r)$ for all $i \in B$, then, almost surely, $x^{(n)} \rightarrow r$ as $n \rightarrow \infty$.

Proof. That convergence occurs with positive probability follows from Lemma 2.3, and the behaviour of $c_{i}^{(n)}$ for $i \in B$ follows from Lemma 2.4 .

For $i \notin S \cup B$, for which $N_{i}(r) / H(r) \rightarrow 0$ as $n \rightarrow \infty$ on $x^{(n)} \rightarrow r$, the same argument as in Lemma 2.4 shows that $c_{i}^{(n)} / n^{\alpha} \rightarrow 0$ for any $\alpha>0$, from which it follows that $f_{i}\left(x^{(n)}\right) / n^{\alpha} \rightarrow 0$ as $n \rightarrow \infty$ if $\alpha>\max _{j \in B}\left(N_{j}(r) / H(r)\right)-2$, which implies that, almost surely, $i$ is visited only finitely many times.

The last part is also a consequence of Lemma 2.3.

Theorem 2.3. In the 'true twins' case, let $G_{0}$ contain a clique $S$ with outer boundary $B$ such that $S$ is not contained in a larger clique, and let $q$ be a stable equilibrium with support $S$. Then, with positive probability, for some stable equilibrium $r \in B_{V_{q}}(\varepsilon)$ with support $S$,

1. $x^{(n)} \rightarrow r$;

2. for $i \in B, c_{i}^{(n)} / n^{\hat{N}_{i}(r) / \hat{H}(r)}$ converges to a limit in $(0, \infty)$ almost surely;

3. for some (random) time $n, \mathcal{R}_{n, \infty}=S \cup B$.

Furthermore, if there is a stable equilibrium $r$ in the limit set $L(x)$ of $\left(x^{(n)}\right)_{n \in \mathbb{N}}$ with support $S$, then, almost surely, $x^{(n)} \rightarrow r$ as $n \rightarrow \infty$.

Proof. The proofs of Lemmas 2.2 and 2.3 apply in this case as well, with $N_{i}$ and $H$ replaced by $\hat{N}_{i}$ and $\hat{H}$. For Lemma 2.4 , if we define

$$
Y_{i}^{(n)}=\sum_{k=1}^{n} \frac{\mathbf{1}_{\left\{X_{k}=i\right\}}}{c_{i}^{(k-1)}}
$$

as before, then

$$
\mathrm{E}\left(Y_{i}^{(n+1)}-Y_{i}^{(n)} \mid \mathcal{F}_{n}\right)=\frac{\hat{N}_{i}\left(x^{(n)}\right)}{\hat{H}\left(x^{(n)}\right)\left(n+n_{0}\right)}+\frac{\hat{N}_{i}\left(x^{(n)}\right)-\hat{H}\left(x^{(n)}\right)}{\left[\hat{H}\left(x^{(n)}\right)\left(n+n_{0}\right)-1\right] \hat{H}\left(x^{(n)}\right)\left(n+n_{0}\right)},
$$

so we redefine the martingale $M_{i}^{(n)}$ by

$$
M_{i}^{(n)}=Y_{i}^{(n)}-\sum_{k=1}^{n}\left(\frac{N_{i}\left(x^{(k-1)}\right)}{H\left(x^{(k-1)}\right)\left(k+n_{0}\right)}+\frac{\hat{N}_{i}\left(x^{(n)}\right)-\hat{H}\left(x^{(n)}\right)}{\left[\hat{H}\left(x^{(n)}\right)\left(n+n_{0}\right)-1\right] \hat{H}\left(x^{(n)}\right)\left(n+n_{0}\right)}\right) .
$$

The rest of the argument is the same as in the proof of Lemma 2.4.

\section{Acknowledgement}

The authors would like to acknowledge the support of the EPSRC funded Amorphous Computing, Random Graphs and Complex Biological Systems group (grant reference EP/ D003105/1).

\section{References}

[1] Bebeк, G. et al. (2006). The degree distribution of the generalized duplication model. Theoret. Comput. Sci. 369, 239-249.

[2] Benaïm, M. (1997). Dynamics of stochastic approximation algorithms. In Séminaires de Probabilités XXXIII (Lecture Notes Math. 1709), Springer, Berlin, pp. 1-68. 
[3] Benaïm, M. and Tarrès, P. (2008). Dynamics of vertex-reinforced random walks. To appear in Ann. Prob.

[4] Bollobás, B. And Riondan, O. M. (2003). Mathematical results on scale-free random graphs. In Handbook of Graphs and Networks, eds S. Bornholdt and H. G. Schuster, Wiley-VCH, Weinheim, pp. 1-34.

[5] Bonato, A. et al. (2010). Models of on-line social networks. To appear in Internet Math.

[6] Chung, F., Lu, L., Dewey, T. G. and Galas, D. J. (2003). Duplication models for biological networks. J. Comput. Biol. 10, 677-687.

[7] Kumar, R. et al. (2000). Stochastic models for the web graph. In Proc. 41st Annual Symp. on Foundations of Comput. Sci. (Redondo Beach, CA, 2000), IEEE Computer Society Press, Los Alamitos, CA, pp. 57-65.

[8] Pemantle, R. (1988). Random processes with reinforcement. Doctoral Thesis, Department of Mathematics, Massachusetts Institute of Technology.

[9] Pemantle, R. (2007). A survey of random processes with reinforcement. Prob. Surveys 4, 1-79.

[10] Raval, A. (2003). Some asymptotic properties of duplication graphs. Phys. Rev. E 68, 066119, 10 pp.

[11] Volkov, S. (2001). Vertex-reinforced random walk on arbitrary graphs. Ann. Prob. 29, 66-91. 